\title{
Ternary Iron Phase Diagram Updates
}

Updates on multicomponent iron phase diagrams are published regularly in this journal. These brief reviews include new phase diagrams of systems that are investigated for the first time, as well as previously investigated systems on which additional information or substantial revision of existing data has become available.

This issue carries updates on Al-C-Fe, Al-Fe-Mo, Al-Fe-Ni, C-Co-Fe, Co-Fe-Gd, Cu-Fe-Mn, $\mathrm{Cu}-\mathrm{Fe}-\mathrm{Sn}, \mathrm{Cu}-\mathrm{Fe}-\mathrm{Zn}$, Dy-Fe-Ge, Dy-Fe-Sb, Fe-Gd-Ge, Fe-Si-Ti, and Fe-Ti-Y ternary systems.

This work was supported by the Indian Institute of Metals, Calcutta.

V. Raghavan

Editor

Phase Diagrams of Ternary Iron Alloys

Parts 1, 2, 3, 5 and 6 